\title{
Complete Neurological Recovery In A 3 Year Old Presenting With Posterior Reversible Encephalopathy Syndrome Due To Idiopathic Hypertension - A Case Report
}

\author{
Dr. Nandita Bhalla ${ }^{1 *}$, Dr. Shrikiran Aroor ${ }^{2}$ \\ ${ }^{1}$ Intern, Kasturba Medical College, Manipal \\ ${ }^{2}$ Professor, Kasturba Medical College, Manipal
}

\begin{abstract}
Posterior reversible encephalopathy syndrome is an acute neurological illness presenting with clinical symptoms and distinctive MRI findings. Symptoms include headaches, seizures, altered consciousness as well as visual impairment. PRES is always accompanied by peculiar radiological findings of edematous change affecting the rear cerebral area. It commonly occurs in settings where patients are undergoing hypertensive crisis, or there is the use of steroids, calcineurin inhibitors, in the nephritic state or end-stage renal disease. The management includes treating the underlying cause and symptomatic therapy. However, due to relatively fewer pediatric reports, its management isn't specific and rather based on experience. Our patient is a 3-year-old male, who presented with hypertensive crisis and MRI findings confirmed it to be a case of PRES. He was managed with a combined regime of antihypertensive and steroids which lead to complete neurological recovery and resolution of PRES. There are a scarce number of case reports on the use of steroids for the treatment of vasogenic oedema in children.
\end{abstract}

Key Words: Posterior reversible encephalopathy syndrome, Hypertensive crisis, Paediatrics, Management

\section{INTRODUCTION}

Posterior reversible encephalopathy syndrome is an acute neurological illness presenting with clinical symptoms and distinctive MRI findings ${ }^{1}$. It is often associated with patients who have hypertension or renal insufficiency. Symptoms are commonly seizures, altered consciousness, headache as well as visual impairment ${ }^{2}$. Our patient presented with hypertensive crisis, his symptoms and MRI findings were correlated and he was diagnosed as PRES. Antihypertensive followed by steroids as treatment resulted in complete neurological resolution and stable blood pressure. 


\section{CASE DESCRIPTION}

A 3-year-old boy presented to the pediatric emergency triage with symptoms of sudden onset abdominal pain since 3 days associated with fever since one day. Past history was unremarkable. His birth history was uneventful, development was normal and immunization was age appropriate. Child's maternal and paternal grandfathers are known cases of hypertension.

On examination, child was conscious, but irritable and not following basic verbal commands. His height was $101 \mathrm{~cm}$ (85th centile), weight was $13.6 \mathrm{~kg}\left(\mathrm{~b} / \mathrm{w} 15\right.$ th and $50^{\text {th }}$ centile) and head circumference $49 \mathrm{~cm}$ (mean and- 2 S.D) suggesting normal growth. However, his B.P was 130/90 (100th centile) in the right upper limb and 140/90 in the left lower limb. Other vital signs were normal but pallor was present, with no icterus, cyanosis, clubbing, lymphadenopathy or edema. His GCS was 13/15.In Central nervous system examination bilateral plantar reflex was extensor, but rest of the neurological examination was normal. Other systems did not reveal any abnormalities.

Blood investigations showed low $\mathrm{Hb}-10.6 \mathrm{~g} / \mathrm{dl}$ with low MCV (66.6fl) and high RDW (16.8\%) suggesting iron deficiency anemia. WBC: $19.8 \times 10^{3} / \mu \mathrm{L}$. Platelet count: 569x103/ $\mu \mathrm{L}$. Rest of the blood counts were normal and ESR was $11 \mathrm{~mm} / \mathrm{hr}$. Serum electrolytes, blood sugar, renal and liver function tests, as well as urine microscopic findings were within normal limits. Serum CRP (0.64mg/dl), compliment C3(180mg/dl), and C4(32.3mg/dl), ASO titre and ANA profile did not show abnormal results. USG abdomen, renal doppler, CT angiogram of renal vessels, 2D ECHO of heart, 24 hour urine levels of VMA (3.2 mg/24 HRS) and HVA (20.1 mg/g creatinine), metanephrine and thyroid function test ( T4- $9.81 \mu \mathrm{g} / \mathrm{dl}$ and TSH- 1.4 $\mu \mathrm{IU} / \mathrm{ml}$ ), renin/aldosterone ratio - done as a part of HTN work up were within normal limits. Patient was extensively investigated but underlying cause of hypertension was not apparent.

Imaging: MRI BRAIN : Intra axial ill- defined patchy altered signal intensities are seen in subcortical and deep white matter of left frontal, bilateral parietal lobes, B/L basal ganglia, posterior limb of internal capsule, right superior and middle cerebellar peduncles and adjacent right cerebellar hemisphere with mild edema.

EEG displayed focal disturbance of electrical function over right temporo- parieto occipital region. CECT brain was normal.

CSF sample for microscopy showed high total WBC count (45 cells/cumm),RBC 100 cells/cumm. CSF glucose $(45 \mathrm{mg} / \mathrm{dl})$ and protein $(36 \mathrm{mg} / \mathrm{dl})$ were normal. CSF culture was sterile, CSF for viral studies and tuberculosis were also negative.

RT-PCR report for COVID 19 from nasopharyngeal and throat swabs were also negative. 
Treatment: The MRI findings were consistent with a diagnosis of atypical posterior reversible encephalopathy syndrome due to hypertensive crisis, thus, he was admitted and started on nifedipine. As hypertension gradually improved but persisted, furosemide and enalapril were added. CSF analysis showed lymphocytic pleocytosis and EEG done showed focal disturbance hence he was started on I.V antiobiotics (Cefotaxime\& Amikacin) and as a prophylactic measure for seizures, on levetiracitam. He became afebrile a day after starting antibiotics. Ten days after admission, the patient partially recovered and was discharged with advice to continue antihypertensives and come for regular follow ups.

However, he presented to paediatric emergency triage after 10 days of discharge, with symptoms of fever of 6 days duration with drowsiness of 3 days duration following 2 episodes of partial complex seizures (on right side).Hypertension was persisting with BP measuring 130/86 mmhg in right upper limb. CNS examination revealed limitation of gaze with left sided 3rd cranial nerve palsy, right sided 6th C.N palsy and facial deviation to left. On motor examination left upper and lower limbs were hypotonic. Reflexes were normal. Cerebellar exam showed nystagmus in left eye and truncal ataxia. Other systems were within normal limits. Blood Counts, renal function tests, electrolyte levels, LFT and urine exam reports did not show abnormal results. Repeat RT-PCR for COVID-19 and COVID-19 antibodies were negative. On admission, his dose of prazocin, nifedipine, levetiracitam were optimised. MRI of brain showed mild reduction in extent of supratentorial lesions and increase in extent of infratentorial lesions as compared to previous study.

MR spectroscopy- Revealed reduced NAA:Choline ratio and mildly increased lipid lactate peak in right basal ganglia with 144 msecTE. Metabolite levels in present study did not suggest possibility of CNS lymphoma/metastasis. Post MRI findings of recurrent atypical PRES he was started on Injection methyl prednisolone at $30 \mathrm{mg} / \mathrm{kg} /$ day for 3 days, followed by oral prednisolone for next 4 weeks. It was decided to stop prazocin, nifedipine and to add metoprolol plus amlodipine. Child became afebrile and recovered neurologically over next 72 hours. His gaze improved and he was able to walk without support. He was discharged with advice to continue Levetiracetam,Prednisolone, Amlodipine, Metoprolol, with B.P monitoring. He was reviewed after 4 weeks when he had remained seizure free and recovered completely neurologically. His blood pressure was within normal limits. Repeat MRI revealed intra axial ill-defined FLAIR hyper intensities with no post contrast enhancement. 

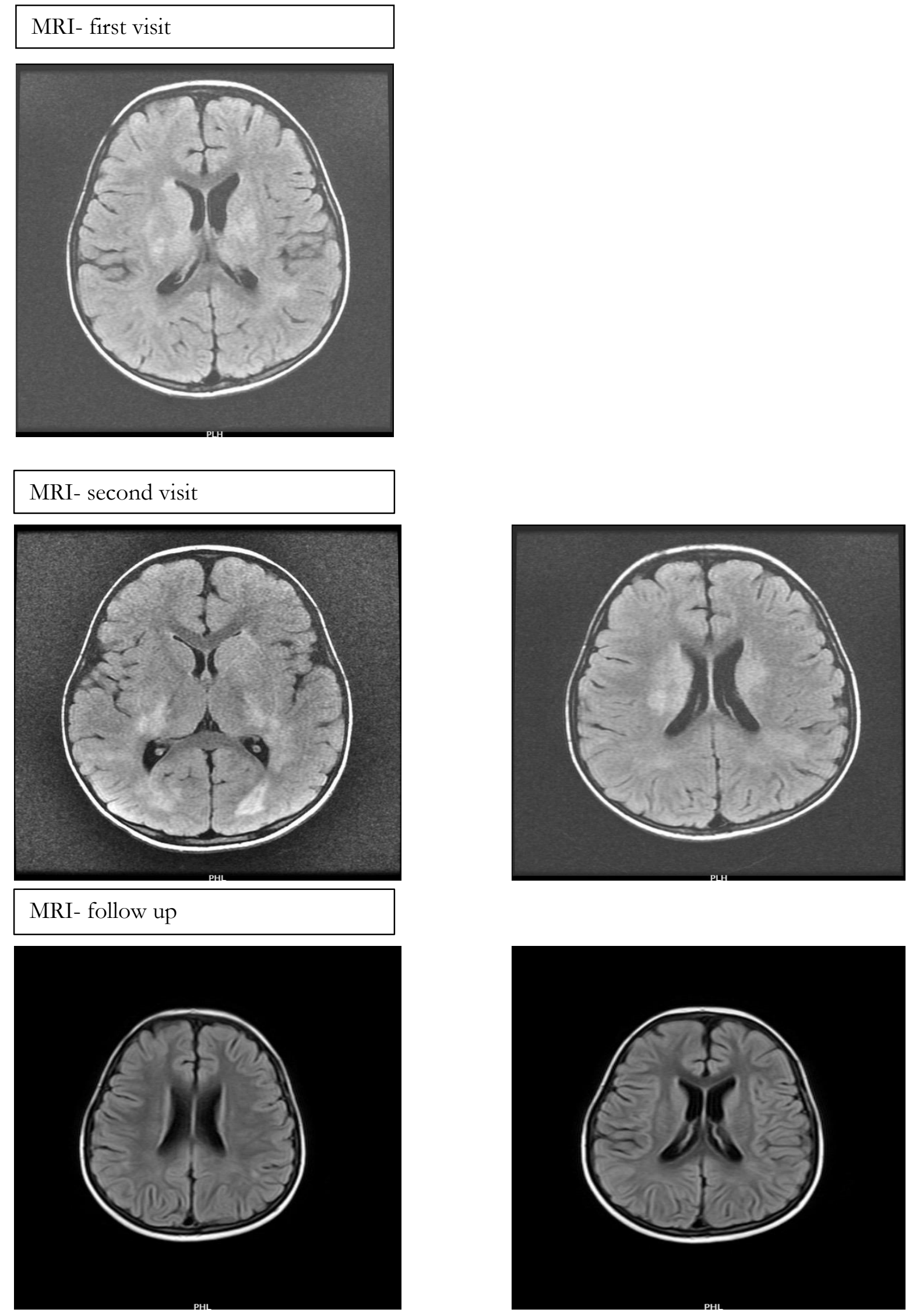


\section{DISCUSSION}

Posterior Reversible Encephalopathy syndrome is an acute neurological syndrome characterized symptomatically by headaches (50\%), altered consciousness (50-80\%), visual disturbances (33\%), seizures (60-70\%) and radiological changes which can resolve. However, if left untreated it can be fatal and not all cases are reversible ${ }^{1,3}$ It can occur in many settings, the most common being hypertensive crisis ${ }^{2}$, other factors include use of steroids, calcineurin inhibitors ,nephrotic state or end stage renal disease ${ }^{4}$. Our patient is a young male with hypertensive crisis.Alongside symptoms, the diagnosis of PRES is always accompanied by peculiar radiological findings of edematous change affecting the rear cerebral area ${ }^{5}$. His MRI findings fall into the atypical locations ${ }^{6}$. With practice of increase in usage of MRI, PRES has become more recognisable to physicians ${ }^{7}$. A broad spectrum of clinical and radiologic patterns have emerged in reports on adult PRES. However, due to relatively fewer pediatric reports, its management isn't specific and rather based on experience. It has been known that PRESinducing disorders can vary with age and the clinical course of PRES might differ among different age groups ${ }^{8}$.

Management starts with investigating the underlying cause and looking for any signs of end organ damage ${ }^{9}$. Our patient was extensively investigated but cause for Hypertension could not be ascertained. There were no signs of end organ damage, thus supportive treatment was initiated. Hypertension is managed by antihypertensives. Hypertensive urgencies, like in our patient, are treated with a mix of oral and parenteral drugs which vary from case to case ${ }^{10,11}$.It is generally advised to gradually lower the B.P, an approach recommended by T.flynn and tullus $^{12}$ advises that BP should be reduced by $25 \%$ of the planned BP reduction over first 8 $12 \mathrm{~h}$, a further $25 \%$ over the next $8-12 \mathrm{~h}$, and the final $50 \%$ over the $24 \mathrm{~h}$ after that, while stein and ferguson ${ }^{13}$ have added that often, therapy has to be augmented to achieve adequate B.P control within first 48 hours. Some known oral drugs effective in treatment are nifedipine, furosemide, prazosin, metoprolol, amlodipine ${ }^{10,11}$ and short acting parenteral drugs include enalapril $^{13}$ among others, all of which were used sequentially in our patient. This changes the systemic vascular resistance and vascular compliance leading to reduction in hypertension ${ }^{14}$ which is an essential part of treatment in PRES ${ }^{15}$. His follow up MRI showed partial resolution of abnormal neuroimaging. If PRES recurs, it does so with new clinical and radiologic features ${ }^{16}$. Our patient revisited with new symptoms for which his antihypertensive regimen was altered and prednisolone was started. Steroids are known to be used for treatment of vasogenic edema ${ }^{17}$ in PRES $^{18,19}$ but are also well established offensive agents that might trigger or worsen PRES ${ }^{20}$. Keeping that in mind, he was monitored carefully, followed up closely and after 4 weeks, he had remained symptom free, had stable B.P and had complete neurologic recovery on follow up with resolution of findings in MRI brain. His dose of oral prednisolone was tapered progressively and eventually stopped. Our report showcases the effectiveness of a combined regime of antihypertensives and steroids for complete resolution of PRES in a child. 


\section{LESSONS LEARNT}

- Inclusion of steroids as treatment of vasogenic edema due to PRES with proper monitoring in paediatric population, since they are also a known risk factor for causing PRES.

- Effectiveness of a collective regime of steroids and antihypertensives for complete neurological recovery in PRES presenting as hypertensive crisis in young paediatric population.

- Increasing awareness regarding regime of treatment of PRES due to idiopathic hypertension in paediatric population.

\section{REFERENCES}

1. Hinchey J, Chaves C, Appignani B, et al. A reversible posterior leukoencephalopathy syndrome. New England Journal of Medicine. 1996;334(8):494-500.

2. McCoy B, King M, Gill D, Twomey E. Childhood posterior reversible encephalopathy syndrome. European Journal of Paediatric Neurology. 2011;15(2):91-94. doi:10.1016/j.ejpn.2010.10.002

3. Fugate JE, Rabinstein AA. Posterior reversible encephalopathy syndrome: clinical and radiological manifestations, pathophysiology, and outstanding questions. The Lancet Neurology. 2015;14(9):914-925. doi:10.1016/S1474-4422(15)00111-8

4. Yamada A, Ueda N. Age and gender may affect posterior reversible encephalopathy syndrome in renal disease. Pediatric Nephrology. 2012;27(2):277-283. doi:10.1007/s00467-011-1974-y

5. Bartynski WS, Boardman JF. Distinct Imaging Patterns and Lesion Distribution in Posterior Reversible Encephalopathy Syndrome. Am J Neuroradiol. 2007;28(7):1320. doi:10.3174/ajnr.A0549

6. Li K, Yang Y, Guo D, Sun D, Li C. Clinical and MRI Features of Posterior Reversible Encephalopathy Syndrome With Atypical Regions: A Descriptive Study With a Large Sample Size. Frontiers in Neurology. 2020;11:194. doi:10.3389/fneur.2020.00194

7. Donmez FY, Basaran C, Kayahan Ulu EM, Yildirim M, Coskun M. MRI Features of Posterior Reversible Encephalopathy Syndrome in 33 Patients. Journal of Neuroimaging. 2010;20(1):22-28. doi:10.1111/j.1552-6569.2008.00306.x

8. Siebert E, Bohner G, Endres M, Liman TG. Clinical and Radiological Spectrum of Posterior Reversible Encephalopathy Syndrome: Does Age Make a Difference? - A Retrospective Comparison between Adult and Pediatric Patients. PLOS ONE. 2014;9(12):e115073. doi:10.1371/journal.pone.0115073

9. Onder AM, Lopez $R$, Teomete U, et al. Posterior reversible encephalopathy syndrome in the pediatric renal population. Pediatric Nephrology. 2007;22(11):1921-1929. doi:10.1007/s00467-007-0578-z

10. Rao G. Diagnosis, epidemiology, and management of hypertension in children Pediatrics. 2016;138(2). 
11. Seeman T, Hamdani G, Mitsnefes M. Hypertensive crisis in children and adolescents. Pediatric Nephrology. 2019;34(12):2523-2537.

12. Flynn JT, Tullus K. Severe hypertension in children and adolescents: pathophysiology and treatment. Pediatric Nephrology. 2009;24(6):1101-1112.

13. Stein DR, Ferguson MA. Evaluation and treatment of hypertensive crises in children. Integr Blood Press Control. 2016;9:49-58. doi:10.2147/IBPC.S50640.

14. Digne-Malcolm H, Frise MC, Dorrington KL. How Do Antihypertensive Drugs Work? Insights from Studies of the Renal Regulation of Arterial Blood Pressure. Frontiers in Physiology. 2016;7:320. doi:10.3389/fphys.2016.00320.

15. Fischer M, Schmutzhard E. Posterior reversible encephalopathy syndrome. Journal of Neurology. 2017;264(8):1608-1616.

16. Komur M, Delibas A, Arslankoylu AE, Okuyaz C, Kara E. Recurrent and atypical posterior reversible encephalopathy syndrome in a child with hypertension. Ann Indian Acad Neurol. 2012;15(3):208-210. doi:10.4103/0972-2327.99721.

17. Adukauskienè D, Bivainytè A, Radavičiütè E. Cerebral edema and its treatment. Medicina. 2007;43(2). doi:10.3390/medicina43020021.

18. Parikh NS, Schweitzer AD, Young RJ, et al. Corticosteroid therapy and severity of vasogenic edema in posterior reversible encephalopathy syndrome. Journal of the Neurological Sciences. 2017;380:11-15. doi:10.1016/j.jns.2017.06.044.

19. Meyer M, Niemöller U, Stein T, et al. Positive Effect of Steroids in Posterior Reversible Encephalopathy Syndrome. Case Reports in Neurology. 2019;11(2):173-177. doi:10.1159/000500410.

20. Chen T-H. Childhood Posterior Reversible Encephalopathy Syndrome: Clinicoradiological Characteristics, Managements, and Outcome. Frontiers in Pediatrics. 2020;8:585. doi:10.3389/fped.2020.00585. 